\title{
What day is today? An inquiry into the process of time orientation
}

\author{
ASHER KORIAT and BARUCH FISCHHOFF \\ Hebrew University, Jerusalem, Israel
}

\begin{abstract}
In each of the 6 work days of a 2-week period, approximately equal numbers of passers-by were presented with the question "What day is today?" The experiment took place in Israel, using a total of 273 Ss. Average RT for the production of a correct response revealed a clear curvilinear relationship with the ordinal position of the day of the week, with longest RTs obtained with midweek target days. Errors revealed a similar curvilinear relationship, being most frequent in the middle of the week. These results are discussed in terms of the utilization of temporal landmarks (e.g., the Sabbath) in the process of time orientation. Additional data regarding the distribution of errors and Ss' introspective reports are presented and speculations regarding the mechanism involved are offered.
\end{abstract}

What day of the week is today?

Few theoretical discussions of cognition are pertinent to analysis of how people arrive at the answer to this question. The response appears to be too immediate to allow direct examination of the intervening process, e.g., through reliance on introspective reports. Nonetheless, such questions of time orientation are so basic to behavior that the mechanism involved deserves theoretical and experimental consideration.

One indirect approach involves generating hypotheses regarding the day retrieval process (DRP) from which testable predictions may be derived. Norman (1970) has recently suggested the possible importance of temporal "landmarks" in the answering of queries such as "Where were you on the night of January 8, 1971?" The respondent to such a query might first locate a significant event in the neighborhood of the target date and then use it as an anchor from which a systematic search for local detail proceeds. Temporal landmarks are characterized by their prominence and accessibility in memory. Norman suggests that they are likely to be quite idiosyncratic. One hypothesis is that the DRP is also organized around such landmarks. Intuitively, weekends are likely to constitute obvious landmark candidates in this case. Since the weekends are consensual landmarks, their effect on the DRP may be manifest across individuals, facilitating analyses that would be extremely difficult with more idiosyncratic landmarks.

The central hypothesis of the present study is that the ease of answering a question about a point in time is a function of its distance from the operative landmark. More specifically, it is hypothesized that (1) the latency of correct day retrieval responses and (2) the frequency of erroneous responses will increase with increasing distance of the target day from the closest weekend. Thus, Ss will take longer and make more errors when asked the question opening this article in the middle of the week.

A second general hypothesis is that time orientation tends to rely more heavily on past anchors than on future anchors and that a past anchor will more effectively assist time orientation than a future anchor at a comparable objective time distance. Thus, it is predicted that the curvilinear relationship between day retrieval difficulty and day of week mentioned above will be skewed toward the forthcoming weekend. Although asymmetries in the subjective perception of past and future events have been studied for both short-range (seconds) and long-range (years) periods (Stevens, 1957; Ekman \& Lundberg, 1971), little information is available regarding the time periods under consideration in the present study. Despite the existence of some supporting results in Cohen (1967) and Ekman and Lundberg (1971), this hypothesis is offered for the moment on purely intuitive grounds.

The above analysis surely does not describe all types of DRP. Consider a reader for whom the question "What day is today?" has arisen prior to reading the first line of this article. Rather than trying to identify the day anew, he is likely to search for his own previous response. A relevant distinction might be made between two types of DRPs: meter reading and meter setting. The prepared reader consults a "meter" that he has previously set. The unprepared reader initiates a search process that results in setting a meter. Meter setting may be seen as a problem-solving process that utilizes information irrelevant to meter readers, such as judged distance from a landmark. The above two hypotheses pertain to meter setting. With regard to meter reading, however, there is no reason to expect any relationship between ease of retrieval and the particular target day.

Apart from the weekend, Ss may utilize secondary landmarks during the week that are determined by their own personal schedules and that are likely to be highly idiosyncratic. In the present study, secondary landmarks were solicited from Ss. An attempt is made to examine the extent to which their effect on the DRP parallels that of the weekends.

This experiment was conducted with Jewish students in Israel. An understanding of the structure of the Israeli week is crucial to interpretation of the findings. A 6-day 


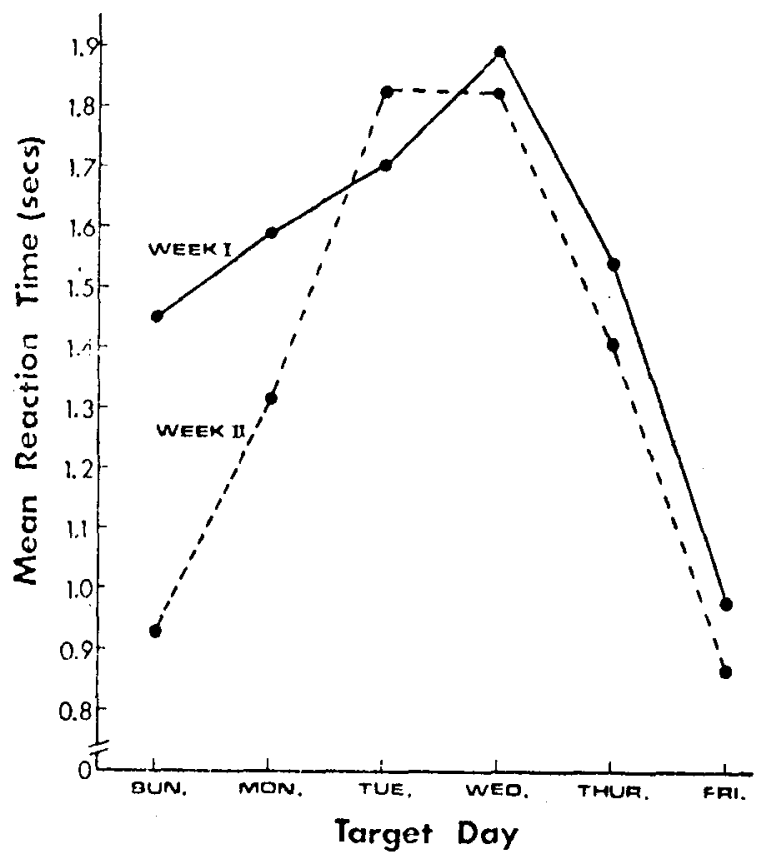

Fig. 1. Mean RT for correct day identification as a function of target day.

work week is virtually universal in Israel. Saturday (the Sabbath) is a rest day, with all commerce, industry, services, and public transportation ceasing on Friday in midafternoon.

\section{METHOD}

Two hundred and seventy-three Ss participated in the present investigation. They were recruited by the $\mathrm{E}$ at various points on the Hebrew University campus between the hours of 9:00 a.m. and 10:00 a.m. each weekday morning of 2 consecutive weeks (134 in Week I and 139 in Week II). Passers-by were asked to participate in a short psychological experiment. Those who agreed became Ss (the refusal rate was approximately $25 \%$ ). Most Ss were students from various University departments. An attempt was made to obtain equal numbers of Ss each day in the given time period, although this was not always possible. Over the 12 experimental days, the number of Ss varied from 20 to 24. The E, an attractive female undergraduate, was not informed of the purpose of the experiment until after the completion of the data-gathering stage. ${ }^{1}$ The experiment was conducted in Hebrew.

Each S was asked two questions: (1) "Name a country beginning with the letter "gimmel" (approximately "g"); (2) "What day of the week is today?"

The time taken by $S$ s to answer each question was measured by the E using a clearly revealed stopwatch. Retrieval time (RT) was measured from the end of each question to the production of a correct answer. In the event of an incorrect answer, the $\mathrm{E}$ made no response and waited for a correct answer. RT for both tasks (RT country and RT day) as well as errors were recorded. In the event of an error, RT was noted both until the error (RT error) and until the correct response (RT).

One reason for the introduction of the first question was to accustom Ss to the timing and questioning procedure before the second, critical question. After completing the retrieval tasks, Ss were asked the following three questions: (1) "How did you know that today is__?" (2) "Has the question 'What day is today' arisen previously this morning?" (Ss who answered affirmatively are referred to as "repeaters.") (3) "What is the most prominent day of your week?" (If a $S$ found the question unclear, he was asked to cite a day that was salient from any point of view, particularly liked or disliked, overloaded, etc. Ss who responded with either Friday or Saturday (the Sabbath) were asked to provide an additional day.)

\section{RESULTS}

\section{Retrieval Time}

The mean RT required to identify the day of the week is presented in Fig. 1 for Week I and Week II separately. In both weeks, the same pattern emerges: The closer the day is to the Sabbath, the natural anchor of the week, the shorter the RT. In the middle of the week (Tuesday and Wednesday), Ss take almost twice as long to retrieve the correct name of the day than on either Friday or Sunday. Because of the similarity of the patterns, the following statistical analyses will be based on the 2 weeks combined.

A one-way analysis of variance for day yielded $F(5,267)=4.018$, significant at the .005 level. The RT means (in seconds) for Sunday, Monday, ..., respectively, were $1.174,1.451,1.770,1.850,1.473$, and .930 . The respective standard deviations were .658 , $1.179,1.800,1.296,1.094$, and .537 .

Thus, the first hypothesis is supported. The farther the S's target is from the closer temporal anchor, the longer it takes to retrieve the tag associated with that target. A test for quadratic trend in RT over days (Winer, 1971, p. 179) yielded $F(1,267)=21.885$, $\mathrm{p}<.001$.

The second hypothesis is that a past temporal anchor more effectively assists time orientation than a future anchor at a comparable objective time distance. The RT pattern obtained for Week I appears to be in line with this hypothesis: The highest RT was obtained on Wednesday. For Week II, however, practically the same RT means were obtained for Tuesday and Wednesday. Possibly the structure of the Israeli week does not allow a proper test of this hypothesis with the present data. Friday, a half-holiday, seems to be psychologically very different from Sunday and appears to constitute a semianchor for many individuals. Supporting this suggestion is the fact that when asked to specify the most salient day of the week, the first response offered was Friday for 61 Ss and Saturday for 56 Ss. We note also that the RT for Sunday is significantly longer than that for Friday. A more adequate test of this hypothesis will have to be conducted in a situation where the psychological middle of the week is more readily identified.

An alternative interpretation of the RT pattern would be that it reflects a midweek decrement in overall performance due to sluggishness, fatigue, etc. If this were so, the same pattern should obtain in a comparable retrieval test. Response latency in the country name production task ("Name a country which begins with 'gimmel") could serve as a control condition to test this possibility. The RT means for this task were $1.87,3.42$, 
$1.98,2.28,1.77$, and 2.20 for Sunday, Monday, ... respectively. A one-way analysis of variance for day yielded $F(5,267)=0.828$. Thus, this alternative explanation may be rejected.

A second use of the country name reaction time was to provide baseline information on individual differences in RT, a potential covariate for increasing the sensitivity of the above analysis. However, the correlations between response latencies on the two tasks (computed separately for each of the days) were negligible, making covariate analysis pointless.

\section{Errors}

A second index of retrieval difficulty is the number of errors made prior to the correct response. Overall, there were 36 errors made by $33 \mathrm{Ss}$. Three Ss made two errors each prior to the correct response. (Two of these were on a Tuesday and one on a Wednesday.) Figure 2 presents percentage of errors as a function of the day of the week. The pattern obtained is very similar to that shown with RT: 24 errors were made on Tuesday and Wednesday as against 12 on the remaining 4 days of the week. A chi-square analysis performed on the number of erring Ss for each day yielded $\chi^{2}=18.45$, $\mathrm{df}=5$, significant at the .01 level.

A quantitative analysis of errors revealed the following: (a) In 30 of 36 cases the incorrect response was the name of a contiguous day. If incorrect responses were distributed randomly over inappropriate days, only one-third of the incorrect responses would be expected to fall on contiguous days $(\mathrm{z}=6.26, \mathrm{p}<.001$, normal approximation to binomial test). (b) Twenty-eight out of the 36 incorrect responses referred to days earlier in the week than the target day. If errors were randomly distributed, an equal number of errors earlier and later in the week would be expected. The probability of as extreme a distribution as the present is less than .001 $(z=4.50$, normal approximation to binomial test). (c) Out of the five noncontiguous incorrect responses, four referred to Sunday. The day retrieval RT analysis presented in the previous section was repeated eliminating all Ss who had made errors. Virtually the same results were obtained $[F(5,235)=3.045, \mathrm{p}<.01]$.

\section{Meter Reading and Meter Setting}

Repeaters were identified as those reporting that the question "What day is today?" had arisen prior to the experiment. Over all test days, there were 47 repeaters. Their distribution over the days of the week was: Sunday, 5; Monday, 8; Tuesday, 9; Wednesday, 7; Thursday, 7; and Friday, 11.

Over the days of the week, the average RT of repeaters was 1.217 as compared to 1.494 for nonrepeaters $(t=1.97, p<.05)$. Thus, if repeaters are in fact meter readers, it can be concluded that meter reading is quicker than meter setting.

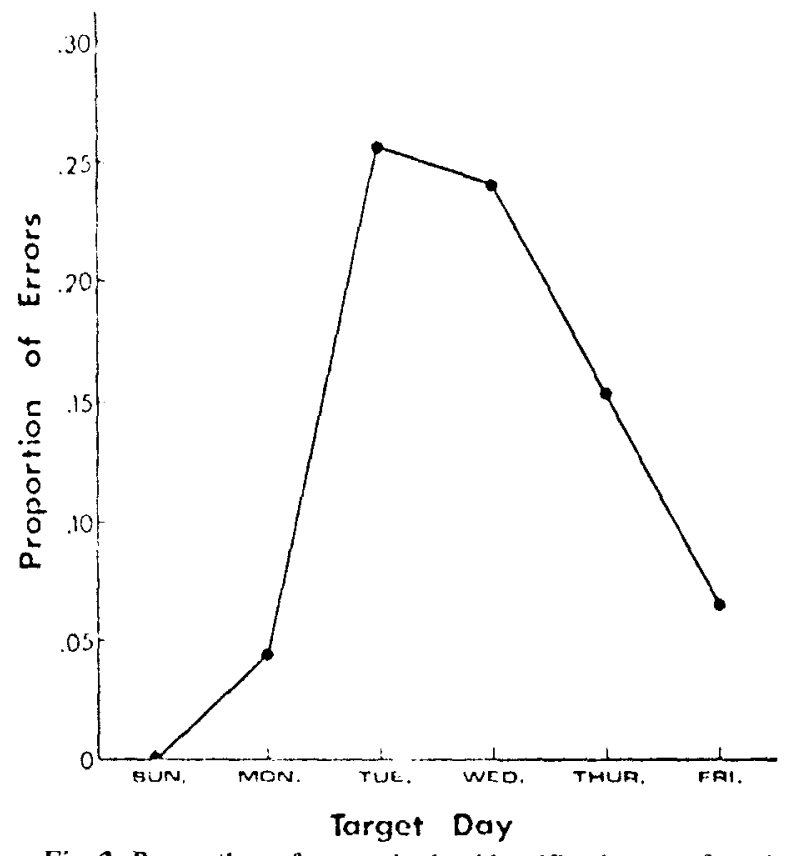

Fig. 2. Proportion of errors in day identification as a function of target day (Week I and Week II combined).

As mentioned above, the landmark-assisted search model pertains to meter setters, not meter readers. With regard to meter setting, shorter latencies are expected the closer the target day is to the Sabbath. With regard to meter reading, however, there is no reason to expect any relationship between RT and the particular target day.

The day by day average RTs for nonrepeaters were as follows: $1.163,1.527,1.842,1.942,1.430$, and .980 . A one-way analysis of variance yielded $F(5,220)=3.494$, $\mathrm{p}<.01$. The respective values for repeaters were: 1.260 , $1.100,1.467,1.174,1.743$, and .773 . A one-way analysis of variance yielded $F(5,41)=1.759$, not significant. Note that the repeaters' average RT is similar to nonrepeaters' RT on Sunday.

\section{The Effect of Idiosyncratic Landmarks}

Although weekends seem to constitute effective consensual landmarks, day retrieval may also be assisted by additional idiosyncratic anchors. The number, strength, and identity of such idiosyncratic anchors may vary from one individual to another and for any individual from time to time. To gain insight into the possible effects of idiosyncratic landmarks, Ss were asked to identify the prominent day in their week (other than Friday or Saturday), a likely candidate for anchor status. If these landmarks affect the DRP in a manner similar to that of the consensual landmarks, then retrieval difficulty should be low for prominent day and should increase with increasing distance from it.

Of the 240 Ss who provided a prominent day, for 38 the experiment took place on that day. (These prominent experimental days were evenly distributed over the days of the week.) Their average RT was 1.211 


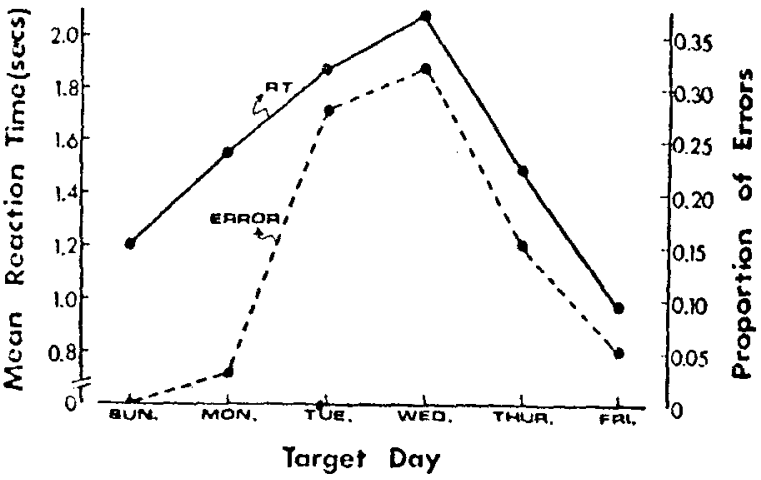

Fig. 3. Mean RT and proportion of errors as a function of target day using only nonrepeater Ss for whom prominent day is other than target day (Week I and Week II combined).

compared to 1.420 for the remaining Ss, a difference that is marginally significant $(t=1.620, p<.10)$. There was no appreciable difference in the error rates for the two groups.

Two-way ANOVAs (target day by prominent day, 6 by 5) were run for RT and errors, using all Ss who reported a prominent day other than Friday or Saturday. Both the effects of target day (as expected) and prominent day on RT were significant and did not interact. The results for prominent day suggest that latency of day retrieval is longest for Ss whose prominent day happens to be on Sunday and, thus, apparently fails to provide an additional midweek anchor. With regard to errors, prominent day yielded no significant effect, but target day (as expected) and the interaction were significant. The latter seems to result from relatively higher error rates when the target day and prominent day are contiguous.

\section{Reexamination of the Asymmetry Hypothesis}

The data presented in Figs. 1 and 2 include both meter readers and Ss whose prominent day fell on the day of the experiment. The inclusion of these two types of Ss tends to obscure the relationship between day retrieval difficulty and distance from the weekend. To obtain a clearer picture of this relationship, the data were reexamined using only nonrepeaters for whom the target day was other than their prominent day. These data are presented in Fig. 3. One noteworthy feature in this figure is that both highest RT and highest proportion of errors are obtained on Wednesday. These presumably "cleaner" data offer stronger support for the asymmetry hypothesis than those presented in Figs. 1 and 2.

\section{Analysis of Introspective Reports}

Answers to the question "How did you know that today is ___?" were classified into five categories: (a) reference to an event the same day (e.g., a class just left or about to start), (b) reference to the previous day (yesterday), (c) reference to the next day (tomorrow), (d) don't know, (e) miscellaneous. The responses given by erring Ss were too idiosyncratic to allow reliable classification. Ignoring erring Ss, the distribution of responses among categories was: (a) 103, (b) 75, (c) 20, (d) 21 , (e) 21 .

The various types of responses were unevenly distributed over the 6 days of the week. The interaction of Type of Response by Target Day was primarily due to the yesterday and tomorrow responses. The distribution of yesterday responses over Sunday, Monday, etc., was $34,18,11,3,5,4$. The corresponding frequencies of tomorrow responses were $0,1,2,0,8,9$. Two conclusions may be readily drawn. First, reference to yesterday is almost four times more frequent than reference to tomorrow $(\mathrm{z}=5.54, \mathrm{p}<.001$, normal approximation to binomial test). Second, in the first 3 days of the week, there were 63 yesterday responses and 3 tomorrow responses, as compared to 12 yesterday responses and 17 tomorrow responses in the last 3 days of the week $\left(\chi^{2}=35.44, \mathrm{df}=1, \mathrm{p}<.001\right)$.

These observations suggest the following: First, the probability of any day of the week serving as a point of reference is a function of its distance from the weekend closest to it. In orienting themselves in time, Ss appear to look backward during the first part of the week and forward during the latter part. Second, time orientation seems to be guided more by the past than by the future, supporting the asymmetry hypothesis: A past point of reference serves as a more effective landmark for time orientation than an equally distant future landmark.

\section{DISCUSSION}

The finding that day retrieval difficulty, as reflected in $\mathrm{RT}$ and errors, increases with increasing distance from the proximal weekend is consistent with the idea that the DRP uses temporal landmarks (e.g., the weekends) that serve as anchors for time orientation. It may be valuable at this point to offer a few speculations regarding the nature of the mechanism by which such landmarks are utilized.

If the DRP is indeed organized around landmarks, the relation between temporal distance from a landmark and ease of orientation must still be accounted for. One possibility is that day retrieval involves a serial scanning process. Consider the following tentative model that resembles the "self-terminating serial search model" proposed by Sternberg in the context of other memory tasks (1969). Day labels are scanned and tested serially, starting from Sunday. In the testing stage, each label is matched against whatever information is available to the individual and either accepted or rejected as the target day name. The search is terminated when an adequate match has been obtained.

Of the results presented above, the following are consistent with this model: (1) the increase in RT for 
correct labeling from Monday to Wednesday; (2) the high proportion of errors referring to days preceding the target day in comparison to errors referring to succeeding days (the labels of these days may have been tested and incorrectly accepted before the target label was encountered); (3) the fact that four out of the five noncontiguous incorrect responses referred to Sunday, resulting perhaps from Ss who jumped at the first response that came to mind.

The decrease in latencies of correct labeling from Wednesday to Thursday to Friday cannot, however, be explained by this simple model. The model could be complicated to accommodate this finding by assuming that the scanning process may begin with either end of the week. Such a modification requires postulation of an additional preliminary orientation mechanism that determines the proximal landmark from which scanning proceeds. Were the choices of landmark random, average RT would be constant over target days.

If the dependence of ease of orientation on the proximity of landmarks is taken as a basic postulate of cognitive functioning, an alternative model may be offered. This model conceptualizes day retrieval as a two-stage process. In the first, a preliminary orientation stage, the general location of the target day in the week is assessed. This may be accomplished by a process similar to Neisser's preattentive evaluation (1967). The decision that the target day is in the beginning, middle, or end of the week narrows the search down to 2 or 3 possible days. In the second stage, one of this restricted set of alternative labels is selected on the basis of more articulate information, such as that mentioned in $\mathrm{Ss}^{\prime}$ introspective reports. According to this model, the curvilinear relationship observed between retrieval difficulty and the ordinal position of the target day is primarily due to the first stage of the search. Determination of the approximate location of the target day is assumed to be easier the closer the target day is to a weekend. On Sunday or Friday, when memory of the past Sabbath or anticipation of the coming Sabbath are vividly present, the second, more analytic stage of the search may be eliminated entirely.

Although it requires further refinement, this two-stage model seems intuitively more faithful than the serial scanning model. It also accounts for one informative finding that can only be awkwardly explained by the first model: the high proportion of errors referring to contiguous days. Twenty out of 26 earlier in the week errors and all seven later in the week errors referred to contiguous days. Ss' introspective reports are of particular interest in this context. Use of yesterday in the earlier part of the week and tomorrow in the later part suggests the availability of information regarding the general location of the target day in the week prior to the search for the actual target day label.

\section{REFERENCES}

Cohen, J. Psychological time in health and disease. Springfield, I11: Thomas, 1967.

Ekman, G., \& Lundberg, U. Emotional reaction to past and future events as a function of temporal distance. Acta Psychologica, 1971, 35, 430-441.

$\mathrm{Neisser,} \mathrm{U}$. Cognitive psychology. New York: Appleton-Century-Crofts, 1967.

Norman, D. N. Remembrance of things past. CHIP-11, University of California, San Diego, 1970.

Sternberg, S., Memory-scanning: Mental processes revealed by reaction-time experiments. American Scientist, 1969, 57, 421-457.

Stevens, S. S. On the psychophysical law. Psychological Review, 1957, 64, 153-181.

Winer, B. J. Statistical principles in experimental design. (2nd ed.) New York: McGraw-Hill, 1971

\section{NOTE}

1. We would like to thank Ofra Razel for her fortitude in collecting and her diligence in analyzing the data in the present study.

(Received for publication May 8, 1973; accepted June 1, 1973.) 